\title{
La réception des poètes latins dans la littérature européenne
}

Susanna Braund

\section{(2) OpenEdition}

Journals

Édition électronique

URL : https://journals.openedition.org/lettre-cdf/1967

DOI : $10.4000 /$ lettre-cdf. 1967

ISSN : 2109-9219

Éditeur

Collège de France

Édition imprimée

Date de publication : 1 mars 2015

Pagination : 40-41

ISSN : 1628-2329

\section{Référence électronique}

Susanna Braund, «La réception des poètes latins dans la littérature européenne », La lettre du Collège de France [En ligne], 39 | mars 2015, mis en ligne le 01 août 2015, consulté le 17 août 2022. URL http://journals.openedition.org/lettre-cdf/1967 ; DOI : https://doi.org/10.4000/lettre-cdf.1967 


\section{La réception des poètes latins dans la littérature européenne}

\section{La signification du choix de la forme métrique dans les traductions européennes de L'Énéide}

L'une des questions primordiales auxquelles doit faire face quiconque veut relever le défi de (re)traduire l'Énéide, c'est le choix de la forme métrique à utiliser dans la langue-cible : le traducteur doit-il, ou ne doit-il pas, chercher une forme qui reproduise l'hexamètre dactylique de Virgile? J'ai voulu indiquer la grande variété des stratégies utilisées dans les traductions écrites en écossais, en anglais, en français, en italien et en russe pendant la vaste période qui va du $\mathrm{XV}^{\mathrm{e}}$ au $\mathrm{xIX} \mathrm{x}^{\mathrm{e}}$ siècles.

Pendant cette longue période nous pouvons répertorier des variantes : couplets héroïques, vers blancs non rimés, «fourteeners ", alexandrins français et russes, mètres des ballades, octosyllabes, ottava rima, anapestes et hexamètres. Ma tâche a donc été d'analyser et d'interpréter le sens idéologique qui anime et inspire ce mélange varié : je me suis proposée de rechercher et d'analyser les paradigmes culturels qui sont évoqués par le choix de chacune de ces formes métriques. II y a, selon moi, au moins deux axes à l'aide desquels le choix d'un mètre de traduction peut être mesuré : l'axe passé/présent, et l'axe domestique/étranger. II s'ensuit que les deux questions correspondantes qui m'occupent sont de savoir, d'un côté, si le mètre choisi par le traducteur utilise l'idiome couramment en usage pour l'épopée ou bien s'il se propose d'offrir des résonances avec l'antiquité; de l'autre, si un tel mètre appartient au vernaculaire propre au traducteur, ou bien s'il est emprunté à une culture étrangère qui est présentée comme le modèle d'une sophistication à assumer.

Le phénomène des traductions partielles : le cas de L'Énéide et des Bucoliques de Virgile

Ma conférence a pris cette fois pour sujet un phénomème signifiant dans les traductions de l'Énéide et des Bucoliques de Virgile. II apparaît clairement que quelques traducteurs seulement traduisent l'intégralité des poèmes, tandis que d'autres ne traduisent qu'une partie, soit d'un livre ou d'un poème, soit d'un extrait plus bref. Par exemple, en France, la traduction des Bucoliques la plus précoce, celle de Guillaume Michel de Tours (1516), est intégrale, mais les traductions suivantes sélectionnent certains passages (Bucolique 1, 1532, Bucolique 2, 1542 et Bucolique 5, 1548).

La quatrième Bucolique était privilégiée dans beaucoup de langues, y compris le danois, le français, le russe et le tchèque. Dans ma conférence, j'ai voulu mener une enquête sur la production des traductions intégrales et partielles de l'Énéide et des Bucoliques faites en Europe, particulièrement en France et en Angleterre. Cette enquête nous offre l'occasion de mettre au jour les tendances à la fois synchroniques et diachroniques. Enfin, après avoir precisé les passages les plus souvent choisis, je me suis demandé pourquoi ces sélections persistaient dans le temps, et à quel moment elles cèdent aux autres sélections puissantes. II résulte de ceci que les facteurs principaux sont les raisons qui motivent le traducteur (ses ambitions), les commandes qu'il a reçues (son protecteur), et le lieu où il a publié sa traduction (le lieu de publication).

\section{Tableaux et spectacles : l'appréciation de Sénèque par les dramaturges européens des XVI et $\mathrm{XVII}^{\mathrm{e}}$ siècles}

À la Renaissance, l'idée du tragique renvoie aux pièces de Sénèque plutôt qu'aux tragédiens grecs. Cela est particulièrement vrai quand les dramaturges se trouvaient en accord avec le mentalité de Sénèque. J'ai émis I'hypothèse que nous pouvons mieux comprendre les tragédies de Sénèque quand nous examinons les drames de la Renaissance qui les ont utilisés comme modèles. Entendu de cette façon, le phénomène de reception fonctionne de façon bidirectionelle, illuminant à la fois le plus ancien et le plus récent. 


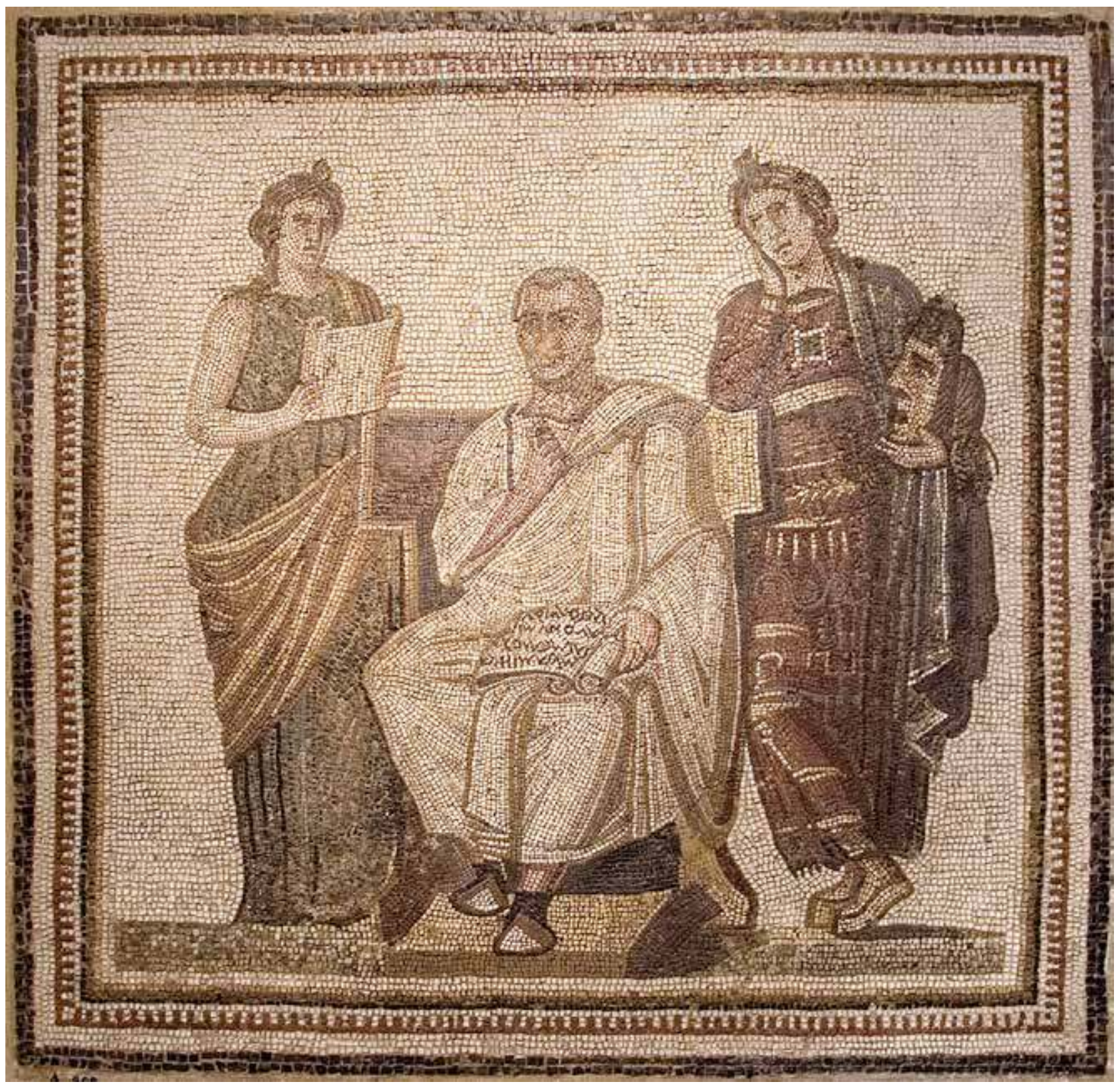

Dans ma conférence, ma methode a été d'examiner deux pièces dans lesquelles les auteurs ont lié les matières prises de Sénèque avec celles prises de Sophocle. L'exemple premier est une pièce française par Robert Garnier, montrée et publiée en 1580, dont le titre est Antigone ou la Piété; l'exemple dernier est une pièce anglaise très populaire par John Dryden et Nathaniel Lee qui s'appelle Oedipus, A Tragedy, qui a été montrée en 1678 et publiée l'année suivante. J'ai voulu montrer comment Garnier a fondé sa conception du rôle d'Antigone sur les tragédies de Sénèque, Oedipe et Les Phéniciennes, et expliquer les éléments dans le drame de Sénèque qui ont tant attiré Garnier. Dans le cas de l'Oedipus de Dryden et Lee, j'ai expliqué comment les auteurs ont vitalisé le drame de Sophocle par l'insertion de la matière empruntée de Sénèque, particulièrement la nécromancie. Il est curieux de voir que les auteurs, dans leur préface, ont critiqué Sénèque et aussi Corneille, mais qu'ils utilisent des éléments importants de ces dramaturges romain et français.

\section{Susanna BRAUND}

- Mme Susanna Braund a été invitée par l'Assemblée des professeurs sur la proposition du Pr John Scheid. - Retrouvez les vidéos de ces conférences sur le site www.college-de-france.fr à la page du Pr John Scheid.
Susanna BRAUND

Professeur à l'University

of British Columbia

(Vancouver) 